\title{
A Case of Systemic Lupus Erythematosus With Spontaneous Intracranial Epidural Hematoma
}

\author{
Rudrajit Paul ${ }^{\mathrm{a}, \mathrm{b}}$, Ramtanu Bandyopadhyay ${ }^{\mathrm{a}}$, Sukamal Santra ${ }^{\mathrm{a}}$, \\ Tushar Kanti Patra ${ }^{\mathrm{a}}$
}

\begin{abstract}
Spontaneous epidural hematoma is a rare clinical entity. We here report the case of a young Indian female presenting with prolonged dull headache consequent to spontaneous epidural hematoma. The patient was diagnosed as systemic lupus erythematosus, an association only rarely reported in literature.
\end{abstract}

Keywords: Systemic lupus erythematosus; Thrombocytopenia; Platelet transfusion

\section{Introduction}

Spontaneous intracranial epidural hematoma is a rare clinical entity. Its association with systemic lupus erythematosus (SLE) is even rarer. We here report the case of a young Indian female presenting with prolonged headache associated with vomiting and new onset abnormal behavior. When the patient was diagnosed as SLE by standard criteria and the blood count revealed thrombocytopenia, she was investigated with CT brain scan which revealed an epidural hematoma. This is the second reported case of spontaneous intracranial epidural hematoma in an SLE patient.

\section{Case Report}

A 16-year-old non-diabetic, non-hypertensive unmarried fe-

\footnotetext{
Manuscript accepted for publication June 15, 2010

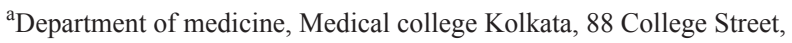
Kolkata-700 073, India

${ }^{b}$ Corresponding author: 15/5, Bose pukur road, Kolkata- 700 039, West Bengal, India. Email: docr89@gmail.com
}

doi:10.4021/jmc2010.07.108e male from West Bengal, India with no addiction, presented with fever with rash and persistent headache for last one month with abnormal behavior for last three days. The rash was maculo-papular with erythema. It increased on sun exposure. They were present mainly in the trunk and neck and were non-itchy. The headache was dull in character, continuous, increased with head movement, associated with vomiting and mainly located in the frontal parts bilaterally. The abnormal behavior was in the form of inappropriate shouting and auditory hallucinations. The patient was admitted elsewhere ten months ago for bleeding manifestations in the form of gum bleeding and menorrhagia for which she had to be given platelet transfusion. But besides thrombocytopenia no other abnormality was found. On examination she revealed mild pallor with oral ulcers (painless), and the papular lesions in the skin mainly on the trunk. Some of the lesions showed necrosis. There were no focal signs in neurological examination. Ophthalmologic examination was normal. There were two mobile discrete lymph nodes in the left axilla, $2 \mathrm{~cm}$ in diameter. The only other finding was mild non-tender splenomegaly. Laboratory tests showed: $\mathrm{Hb} \%$ of $8.5 \mathrm{gm} \%$; TLC of $9,300 / \mathrm{mm} 3$; Platelet $20000 / \mathrm{mm} 3$, ESR of $76 \mathrm{~mm}$ in the first hour; FBS $100 \mathrm{mg} \%$; PPBS $132 \mathrm{mg} \%$; Urea $28 \mathrm{mg} \%$, Cr $0.79 \mathrm{mg} \%$; Urine routine within normal limits (WNL); Chest X-Ray WNL; USG abdomen: nothing abnormal detected except for splenomegaly $(12.7 \mathrm{~cm})$; bilirubin and liver enzyme values-WNL; HIV serology- Non reactive. Thyroid

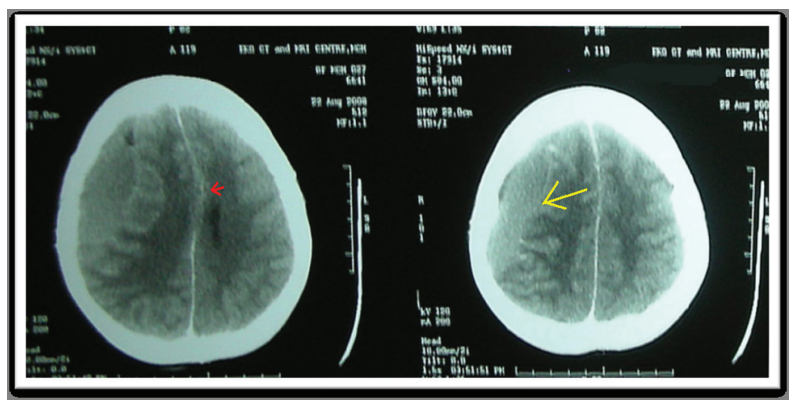

Figure 1. The CT scan of the patient shows the right-sided epidural hematoma (yellow arrow) and the midline shift (red arrow). 
profile was normal; Direct Coombs test was negative; test for malaria double antigen was negative and blood culture on the first day of admission did not grow any organisms. Other tests showed Prothrombin-time as $12 \mathrm{sec}$ (control: $11 \mathrm{sec}$ ); APTT: WNL. Lymph Node biopsy revealed reactive hyperplasia; ANF (ELISA) positive (titer: 3.125); ANF by indirect immunofluorescence (Hep2 method) was positive (1:360) with a speckled pattern. Anti-dsDNA (Hep2) was negative; anti cardiolipin antibody: $\mathrm{IgG}+\mathrm{ve}$. Bone marrow aspirate showed reactive hyperplasia with slightly increased megakaryocytes. Thus this was a case of SLE based on oral ulcer, photosensitive rash, thrombocytopenia and ANA positivity. When the headache was becoming more intense despite the use of analgesics over a period of five days we did CT brain scan which showed right sided epidural hematoma with midline shift (Fig. 1). There was no history of trauma. Thus the case was finally diagnosed as a case of SLE presenting with recurrent thrombocytopenia and spontaneous epidural hematoma. The patient was given platelet transfusion and transferred to the neurosurgery department.

\section{Discussion}

SLE is an autoimmune disease with plenty of tissue binding autoantibodies that cause damage [1]. Thrombocytopenia may be present in $15 \%$ of cases [1]. SLE is diagnosed by the presence of standard criteria $[2,3]$. This is a multi system disease with main involvements in kidney, skin and blood. It is usually present in young adult women and occurs in all populations. However the occurrence of spontaneous extra-dural hematoma in SLE is very rare. The first case was reported from Japan in 1985 where the epidural hematoma was the presenting feature of SLE. Like our case this also presented with headache and vomiting with no history of trauma [4]. Spontaneous intracranial extradural hematoma is a very rare entity with around 19 reported cases to date [5]. Most cases were secondary to infection and the mechanism was thought to be vasculitic rupture of the vessels. In one report of two cases from Singapore [6], the authors have failed to find the cause in one whereas the other was due to coagulation disorder. Very rarely has SLE been linked to spontaneous intracranial hematoma. There is one report of subdural hematoma [7]. Search of literature revealed that this rare complication of epidural hematoma in SLE has been reported only once [4]. The psychotic symptoms can be accounted for by the effect of SLE on brain function [8]. The patient was operated upon with release of blood clots from epidural space.

In conclusion, SLE with spontaneous extradural hematoma is a very rare association. This case shows that any complaint of intractable headache in a patient with SLE and thrombocytopenia should be taken seriously and investigated with a CT brain scan to rule out intracranial pathology.

\section{Abbreviations}

SLE: systemic lupus erythematosus; ANA/ANF: anti-nuclear antibody/factor; APTT: activated partial thromboplastin time; NAD: nothing abnormal detected; WNL: within normal limits; TLC: total leukocyte count; FBS: fasting blood sugar; PPBS: post prandial blood sugar;

\section{Acknowledgements}

Dr. A.K.Banerjee, professor and head, Department of Medicine, Medical College Kolkata.

\section{Conflict of Interest}

None

Financial Support

none

Consent of Patient and Guardian

Taken

\section{References}

1. Hahn BH. Systemic Lupus Erythematosus In Fauci AS, Braunwald E, Kasper DL, Hauser SL, Longo DL, Jameson JL et al Eds. Harrison's Principles of Internal medicine McGraw Hill, NY; 17th edition:2075-83;2008.

2. Tan EM, Cohen AS, Fries JF, Masi AT, McShane DJ, Rothfield NF, et al. The 1982 revised criteria for the classification of systemic lupus erythematosus. Arthritis Rheum1982;25:1271-7.

3. Hochberg MC. Updating the American College of Rheumatology revised criteria for the classification of systemic lupus erythematosus. Arthritis Rheum. 1997 ;40(9):1725.

4. Ishige N, Sunami K, Sato A, Watanabe O, Sakurada M, Ishihara K. [A case of systemic lupus erythematosus associated with spontaneous bilateral epidural hematomas]. No Shinkei Geka 1985;13(3):345-349.

5. Zheng FX, Chao Y. Spontaneous intracranial extradural hematoma: case report and literature review. Neurol India 2009;57(3):324-326.

6. Ng WH, Yeo TT, Seow WT. Non-traumatic spontaneous acute epidural haematoma -- report of two cases and review of the literature. J Clin Neurosci 2004;11(7):791793.

7. Hagen K, Einarsen C, Zwart JA, Svebak S, Bovim G. The co-occurrence of headache and musculoskeletal 
symptoms amongst 51050 adults in Norway. Eur J Neurol 2002;9(5):527-533.

8. Mitsikostas DD, Sfikakis PP, Goadsby PJ. A meta- analysis for headache in systemic lupus erythematosus: the evidence and the myth. Brain 2004;127(Pt 5):12001209. 